\title{
Overcoming of One More Pitfall in Boundary Element Calculations with Computer Simulations of Ion-Selective Electrode Response
}

\author{
Vladimir V. Egorov*, ${ }^{\dagger}$ a \\ ${ }^{\dagger}$ Department of Analytical Chemistry, Belarusian State University, Leningradskaya Str., 14, 220030 Minsk, Belarus \\ ${ }^{\ddagger}$ Research Institute for Physical Chemical Problems of the Belarusian State University, Leningradskaya Str., 14, 220030 Minsk, \\ Belarus
}

Supporting Information

\begin{abstract}
Computer simulations of ion-selective membrane electrodes using diffusion layer models based on finite-differences principle for calculating diffusion processes in both phases and taking into account the local ion exchange equilibrium at the interface are successfully used for clarifying and even predicting the influence of different diffusion factors on several time-dependent characteristics of electrodes. It is shown here that a well-established approach based on the assumption of the constant concentration of the interfering ion in the sample solution fails for solutions containing strongly interfering ions where the concentration of the interfering ion in the boundary layer of the solution can be far lower in comparison with its concentration in the bulk. The limitation is demonstrated by a drastic discrepancy between experimental and calculated curves for the dependence of potential on time. This limitation can be overcome by taking into account the change of the interfering ion concentration in the boundary layer in accordance with the electroneutrality condition. A good agreement between simulation results and experimental data is demonstrated.
\end{abstract}

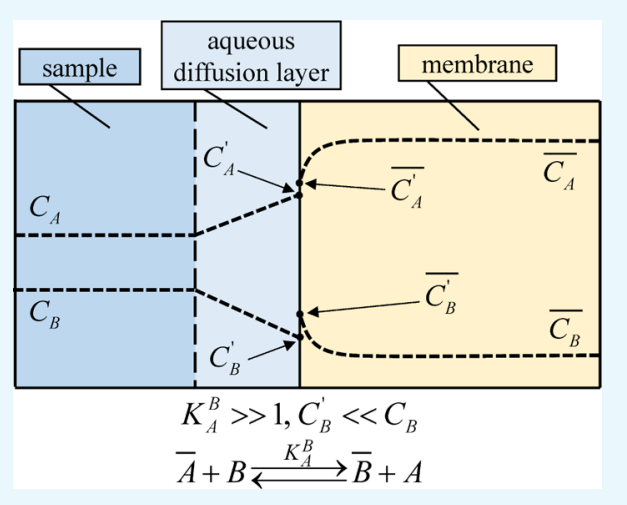

\section{INTRODUCTION}

Ion-selective electrodes or ISEs (the term "ion-selective" reflects their capability to discriminate between the primary and interfering ions) are potentiometric chemical sensors comprising widely used tools for sensing ions in solutions, and having important practical significance for laboratory research and clinical, environmental, and industrial analysis. ${ }^{1,2}$ The obvious advantages of ISEs are low cost of equipment, simplicity of pretreatment (normally no pretreatment is required), operability in turbid and colored media, possibilities of in line and in vivo measurements, nondestructive analysis, continuous monitoring, and so forth. Unlike other analytical techniques, measurements with ISEs provide with data on the activity of the analyte, and in many cases, this is a critical advantage. ISEs are normally classified by the material of the ion-exchange membrane and are divided into glass, crystalline, and liquid membrane (including plasticized polymeric membrane) ones. The last class comprises the most numerous group of ISEs; in this case, the ion-exchange ability of the membrane is achieved by the introduction into the membrane liquid ion exchangers, for example, tetraphenyl borate derivatives as cation exchangers or higher quaternary ammonium salts as anion exchangers. For a long time, it was believed that the response of such electrodes is thermodynamically determined and depends on the Gibbs energy of the process of transfer of the corresponding ion from the solution to the membrane phase, but now it is well known that diffusion processes occurring at the membrane/sample solution inter- face and inside the membrane can have a significant impact on the ISE response, leading, in some cases, to the drastic change of the selectivity, sensitivity, and lower detection limit. ${ }^{3-7}$ Under conditions of diffusion control of the potential, the indicated ISE characteristics strongly depend on the specific experimental conditions, including parameters that are easily adjustable (rate of the solution stirring, concentration) and are usually time dependent. It should be noted that such effects are well known since the 1980s and are discussed in detail in the works of Hulanicki and Lewenstam on the example of solidstate membrane electrodes. ${ }^{8,9}$ In particular, it was shown that if an interfering ion forms a less soluble precipitate, compared to the primary one, its concentration in the near-electrode layer of the solution decreases as compared to the bulk one as a result of reprecipitation. In this case, a concentration gradient arises and the delivery of the interfering ion from the bulk solution to the electrode surface is controlled by the diffusion process. Thus, the experimentally determined selectivity coefficients depend both on the concentration of the interfering ion in the bulk solution and on time. Subsequently, it was shown that diffusion processes also affect the response of ISE with liquid membranes ${ }^{10}$ (in this case, it is necessary to take into account diffusion processes in both phases), and the detected effects have found important practical applications for kinetic

Received: October 24, 2018

Accepted: January 8, 2019

Published: January 18, 2019 
discrimination of strongly interfering ions by reducing the readout time. ${ }^{11,12}$

Because of the considerable difficulties in the experimental study of diffusion processes inside the membrane, on the one hand, and fast progress in the numerical simulation methods, on the other hand, various time-dependent diffusion models of electrode potential have recently been developed. These models have a certain prognostic ability and, in some cases, allow us to evaluate the influence of various factors on the functioning of ISE much faster and cheaper than experimentally.

One of the most frequently used is the dynamic diffusion model proposed by Morf, ${ }^{13}$ based on the use of the finitedifference method and proved to be effective in a number of practical applications. ${ }^{14-19}$ According to this model, the membrane phase and the diffusion layer of the solution are divided into a certain number of conditional layers that are thin enough so that the concentration profiles within each layer can be considered linear, which allows replacing the differentials in the Fick equations describing the diffusion processes inside each of the phases by the ratios of small, but finite values and significantly simplify the calculation algorithm. Therefore, the concentration of any component in any conditional layer in the membrane or in the diffusion layer of the solution, with the exception of boundary layers, at an arbitrary time instant $t+\Delta t$ is easily calculated on the bases of its concentrations in this and two adjacent layers at the previous time instant $t$, and assuming linear diffusion. The equations used for the calculations in the boundary (surface) layers in the case of the ion-exchange membranes can be written as follows

$$
\begin{aligned}
& C_{\mathrm{A}, 0}(t+\Delta t)=C_{\mathrm{A}, 0}(t)+\left[C_{\mathrm{A}, 1}(t)-C_{\mathrm{A}, 0}(t)\right] \frac{D \Delta t}{\delta^{2}} \\
& +\left[\overline{C_{\mathrm{A}, 1}(t)}-\overline{C_{\mathrm{A}, 0}(t)}\right] \frac{\bar{D} \Delta t}{\delta \bar{\delta}} \\
& \overline{C_{\mathrm{A}, 0}(t+\Delta t)}=\frac{C_{\mathrm{A}, 0}(t+\Delta t) \overline{C_{\mathrm{R}}^{\text {tot }}}}{C_{\mathrm{A}, 0}(t+\Delta t)+K_{\mathrm{A}}^{\mathrm{B}} C_{\mathrm{B}}}
\end{aligned}
$$

where symbols with and without a macron refer to the phase of the membrane and to the aqueous phase, correspondingly; $\delta$ is the thickness of the conditional layer; $C_{\mathrm{A}, 0}$ and $C_{\mathrm{A}, 1}$ are the concentrations of the primary ion in the surface (zero) and the first conditional layers; $C_{\mathrm{B}}$ is the interfering ion concentration in the solution; $\overline{C_{R}^{\text {tot }}}$ is the total ion exchanger concentration in the membrane; and $K_{\mathrm{A}}^{\mathrm{B}}$ is the ion exchange constant which is equal to the unbiased selectivity coefficient.

Good prognostic ability of this model has been demonstrated in a number of works. ${ }^{14-19}$ However, it was recently found that the model shows principal limitations that in some realistic scenarios of the electrode operation may lead to the oscillation of the calculated values of potential and even to failure of the calculations. ${ }^{17,19-21}$ Bakker drew attention to the fact that eq 1 is valid only if the concentration of ion $A$ in the zero layer of the membrane is constant. Because, under the nonsteady state conditions this assumption is not fulfilled, an additional term taking into account the change in the concentration of the ion $\mathrm{A}$ must be introduced into eq $1 .^{21}$ Then eq 1 takes the form

$$
\begin{aligned}
& C_{\mathrm{A}, 0}(t+\Delta t)=C_{\mathrm{A}, 0}(t)+\left[C_{\mathrm{A}, 1}(t)-C_{\mathrm{A}, 0}(t)\right] \frac{D \Delta t}{\delta^{2}} \\
& +\left[\overline{C_{\mathrm{A}, 1}(t)}-\overline{C_{\mathrm{A}, 0}(t)}\right] \frac{\bar{D} \Delta t}{\delta \bar{\delta}}-\left[\overline{C_{\mathrm{A}, 0}(t+\Delta t)}-\overline{C_{\mathrm{A}, 0}(t)}\right] \frac{\bar{\delta}}{\delta}
\end{aligned}
$$

Further, solving the system of eqs 2 and 3, we can obtain an analytical solution for calculating the concentrations of the primary ion in the surface layer of the solution.

\section{RESULTS AND DISCUSSION}

We found that Morf's model contains one more pitfall, namely, use of the eq 2 is based on the assumption that the concentration of the interfering ion $\mathrm{B}$ in the near-electrode layer of water is equal to its concentration in the bulk and does not change with time. This assumption is quite acceptable in the case of the operation of ISEs in the presence of highly discriminated interfering ions when the ion-exchange equilibrium is strongly shifted to the left. However, in the case of strongly interfering ions, this assumption is not fulfilled because of the intensive ion-exchange process at the interface. As a result, a drastic discrepancy between the experimental and calculated electrode response is observed (see Figure 1).

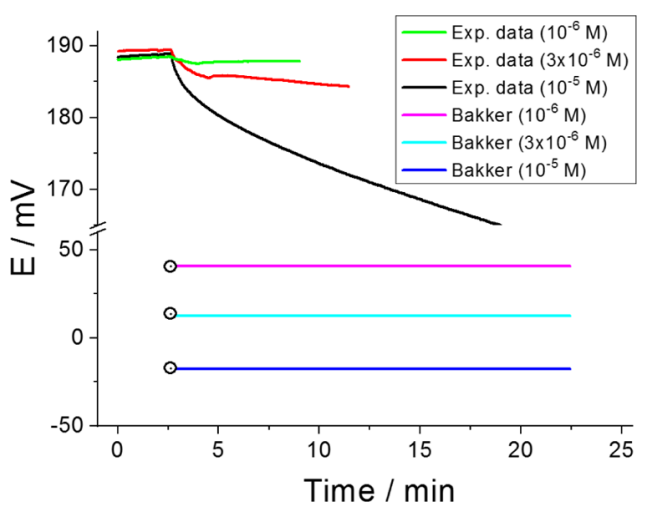

Figure 1. Dependencies of the potential vs time for nitrate SE at different concentrations of picrate in the sample solution (indicated on the legend). The concentration of nitrate in all solutions was $10^{-3}$ M. Simulation parameters: $K_{\mathrm{A}}^{\mathrm{B}}=3.8 \times 10^{5} ; D_{\mathrm{m}}=9 \times 10^{-12} \mathrm{~m}^{2} / \mathrm{s} ; D_{\mathrm{aq}}$ $=1.6 \times 10^{-9} \mathrm{~m}^{2} / \mathrm{s} ; l_{\mathrm{aq}}=25 \mu \mathrm{m}$; and $l_{\mathrm{m}}=400 \mu \mathrm{m}$. Dots in circles represent the potential calculated using the Nikolskii equation.

On this figure, the experimental curves of the dynamics of the potential change for a nitrate-selective electrode in solutions containing different concentrations of picrate (a highly interfering ion) are compared with the corresponding potential on time dependences calculated according to Morf's model improved by Bakker. The choice of picrate ions as the interfering one is due, on the one hand, to the very high lipophilicity of picrate, which ensures their strong interfering effect, and on the other hand, by the guaranteed absence of picrate in salts of the primary ion, nitrate, which is necessary for the purity of the experiment. One can see that calculated potential values are independent of time and correspond to those calculated from the Nikolskii equation. On the contrary, the experimental potential values are time-dependent and the potential drop caused by the injection of picrate is much less than that predicted according to the Nikolskii equation.

The observed discrepancies can be explained by the fact that the concentration of picrate in the near-electrode layer of the 
solution, responsible for the change of potential, is far smaller than the concentration in the bulk solution (due to the intensive ion-exchange process at the interface) and is time dependent. So the eq 2 should be modified as follows

$$
\overline{C_{\mathrm{A}, 0}(t+\Delta t)}=\frac{\overline{C_{\mathrm{R}}^{\mathrm{tot}} C_{\mathrm{A}, 0}(t+\Delta t)}}{C_{\mathrm{A}, 0}(t+\Delta t)+K_{\mathrm{A}}^{\mathrm{B}} C_{\mathrm{B}, 0}(t+\Delta t)}
$$

where $C_{\mathrm{B}, 0}(t+\Delta t)$ is the concentration of the interfering ion in the near-electrode (zero) layer of the solution. It depends on time that can be accounted according to the following considerations. Assuming that the ion exchange is the only way of the mass transfer of ions A and B across the interface while the co-extraction processes can be neglected, the concentration of the co-ion $\mathrm{X}$ in all layers of the sample solution should be equal to its bulk concentration and should not depend on time

$$
C_{\mathrm{X}, 0}(t)=C_{\mathrm{X}, 0}(t+\Delta t)=C_{\mathrm{X}, \mathrm{bulk}}
$$

where $\mathrm{X}$ is the oppositely charged co-ion.

Further, assuming the local electroneutrality condition in the zero layer (as well as in any other layer) is fulfilled for any instant of time, the following equation is valid

$$
\begin{aligned}
C_{\mathrm{A}, 0}(t)+C_{\mathrm{B}, 0}(t) & =C_{\mathrm{A}, 0}(t+\Delta t)+C_{\mathrm{B}, 0}(t+\Delta t) \\
& =C_{\mathrm{X}, \text { bulk }}
\end{aligned}
$$

Thus, the expression for $C_{\mathrm{B}, 0}(t+\Delta t)$ can be written as follows

$$
C_{\mathrm{B}, 0}(t+\Delta t)=C_{\mathrm{B}, 0}(t)+C_{\mathrm{A}, 0}(t)-C_{\mathrm{A}, 0}(t+\Delta t)
$$

The explicit form of the formula for calculating the concentration of the primary ion in the near-electrode layer can be obtained by solving the system of eqs 3,4 , and 7

$$
C_{\mathrm{A}, 0}(t+\Delta t)=0.5\left(a-\frac{1}{K_{\mathrm{A}}^{\mathrm{B}}-1}\left[\sqrt{\left(b+c^{2}\right)}\right]\right)
$$

where

$$
\begin{aligned}
& a=2 C_{\mathrm{A}, 0}(t)-C_{\mathrm{A}, 0}(t) \frac{D_{\mathrm{aq}} \Delta t}{\delta^{2}}+C_{\mathrm{A}, 1}(t) \frac{D_{\mathrm{aq}} \Delta t}{\delta^{2}}+\overline{C_{\mathrm{A}, 0}(t)} \\
& -\overline{C_{\mathrm{A}, 0}(t)} \frac{D_{\mathrm{m}} \Delta t}{\delta^{2}}+\overline{C_{\mathrm{A}, 1}(t)} \frac{D_{\mathrm{m}} \Delta t}{\delta^{2}}+C_{\mathrm{B}, 0}(t) \\
& +\frac{\overline{C_{\mathrm{R}}^{\mathrm{tot}}}+C_{\mathrm{A}, 0}(t)+C_{\mathrm{B}, 0}(t)}{K_{\mathrm{A}}^{\mathrm{B}}-1}
\end{aligned}
$$

$$
\begin{gathered}
b=-4\left(K_{\mathrm{A}}^{\mathrm{B}}-1\right) \\
\overline{C_{\mathrm{R}}^{\mathrm{tot}}}\left(\left[\frac{D_{\mathrm{aq}} \Delta t}{\delta^{2}}-1\right] C_{\mathrm{A}, 0}(t)-\frac{D_{\mathrm{aq}} \Delta t}{\delta^{2}} C_{\mathrm{A}, 1}(t)\right. \\
\left.+\left[\frac{D_{\mathrm{m}} \Delta t}{\delta^{2}}-1\right] \overline{C_{\mathrm{A}, 0}(t)}-\frac{D_{\mathrm{m}} \Delta t}{\delta^{2}} \overline{C_{\mathrm{A}, 1}(t)}\right)
\end{gathered}
$$

$$
\begin{aligned}
& c=\overline{C_{\mathrm{R}}^{\mathrm{tot}}}+\left(1+\frac{D_{\mathrm{aq}} \Delta t}{\delta^{2}}\left[K_{\mathrm{A}}^{\mathrm{B}}-1\right]\right) C_{\mathrm{A}, 0}(t) \\
& +\left(\frac{D_{\mathrm{aq}} \Delta t}{\delta^{2}}-K_{\mathrm{A}}^{\mathrm{B}} \frac{D_{\mathrm{aq}} \Delta t}{\delta^{2}}\right) C_{\mathrm{A}, 1}(t)+\left(K_{\mathrm{A}}^{\mathrm{B}}-1\right) \\
& \times\left(\left[\frac{D_{\mathrm{m}} \Delta t}{\delta^{2}}-1\right] \overline{C_{\mathrm{A}, 0}(t)}-\frac{D_{\mathrm{m}} \Delta t}{\delta^{2}} \overline{C_{\mathrm{A}, 1}(t)}\right)+K_{\mathrm{A}}^{\mathrm{B}} C_{\mathrm{A}, 0}(t)
\end{aligned}
$$

The above formula was obtained for the case when the thicknesses of the surface (zero) layers in the membrane and in the aqueous diffusion layer are equal to each other. If the thicknesses of these layers in the membrane and in the aqueous diffusion layer are different, a somewhat more complicated formula can be obtained.

Besides, if the concentration of the interfering ion in the sample solution is rather small, as it is in the case under consideration, the change of the concentration in the bulk solution $\left(C_{\mathrm{B}}\right)$ due to the uptake by the membrane should be taken into account as well

$$
C_{\mathrm{B}}(t+\Delta t)=C_{\mathrm{B}}(t)+\left[C_{\mathrm{B}, \text { last }}(t)-C_{\mathrm{B}}(t)\right] \frac{D_{\mathrm{aq}} \Delta t}{\delta_{\mathrm{aq}}{ }^{2}} \frac{V_{\text {last }}}{V_{\mathrm{S}}}
$$

where $V_{\mathrm{S}}$ is the sample solution volume; $V_{\text {last }}$ is the volume of the last elementary layer of the aqueous diffusion layer; and $C_{\mathrm{B} \text {,last }}$ is the interfering ion concentration in this layer.

Data presented on Figure 2 illustrate a drastic (up to 5 decimal orders) drop of the concentration of picrate in the

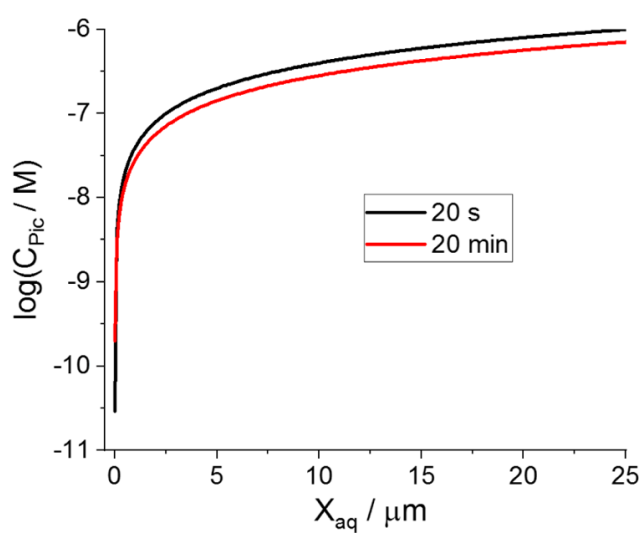

Figure 2. Profiles of picrate ion concentrations in the aqueous diffusion layer calculated for the nitrate-SE operation in a mixed solution of nitrate $\left(10^{-3} \mathrm{M}\right)$ and picrate $\left(10^{-6} \mathrm{M}\right)$. Simulation parameters are indicated in the caption to Figure 1.

near-electrode layer of the sample solution versus its concentration in the bulk. One can see as well that the most pronounced drop of the concentration is observed for the initial times of measurements, while with the increase of time, the picrate concentration in the near-electrode layer of the solution rises. These observations are in full accordance with the experimental results presented on Figure 1. Also finally, one can see that the decrease of picrate concentration in the bulk solution due to its extraction into the membrane should be also taken into account if the measuring time is not too short (the change of the bulk concentration of picrate for 20 min exposition of the electrode in the solution is about $30 \%$ ). 
Data presented on Figure 3 illustrate the predictive ability of the Morf s-Bakker's model modified by us for the case of the

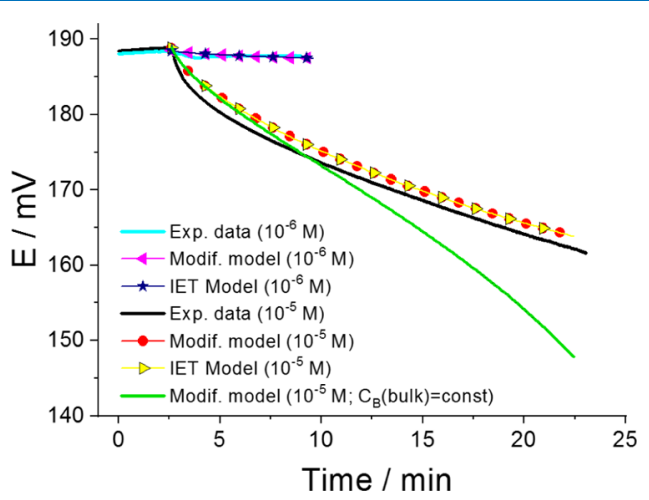

Figure 3. Simulation results in the framework of the Morf's-Bakker's model modified by us and the IET model at low concentrations of picrate in the sample solution (indicated on legend). Simulation parameters are indicated in the caption to Figure 1.

ISE operation in the presence of small concentrations of strongly interfering ions. The calculation results obtained using eqs $3,4,7$, and 12 agree well with experimental data and reflect both far less influence of the interfering ion on the electrode potential than it could be expected from the unbiased selectivity coefficient and the potential dependence on time. Therefore, the distinctive feature of the described model is that, unlike the model proposed by Morf and later improved by Bakker, it takes into account that the concentration of the interfering ions in the near-membrane layer of the solution differs from that in the solution bulk. It should be mentioned here that this idea was expressed earlier by Lewenstam and Hulanicki who proposed to introduce a time-dependent parameter $s(t)$ called the "surface coverage" or "site filling factor" ${ }^{8}$ This parameter was defined as the share of the sites in the surface layer of the membrane occupied by the interfering ions which directly depends on the concentrations of both primary and interfering ions in the surface layer of the solution. ${ }^{8}$ It was shown that the use of this parameter allows one to describe the dependence of the potential on time, including the cases where the primary and interfering ions have different diffusion coefficients in both water and the membrane. However, the explicit form of the equation for calculating this parameter was not proposed at that time, so its use for practical calculations is rather complicated. ${ }^{22}$ The model described by us in this paper does not include the site filling factor $s(t)$ as a modeling parameter. Furthermore, it allows for the calculation of this value, inserting of which into the eq 12 in ref 23 allows us to obtain the same EMF versus time dependence as in the framework of the model described in this paper (see the Supporting Information I for more details).

It should be mentioned here that absolutely the same results using the same modeling parameters can be directly obtained in the framework of the recently proposed interface equilibriatriggered model (IET model) that is based on the separate, step-by-step account of the local equilibrium at the interface and diffusion in the membrane and solution phases. ${ }^{20}$ Namely, each new calculation cycle begins with recalculating the concentrations of components in sufficiently thin surface layers of the membrane and solution, based on the concentrations of these components established at a previous time point as a result of diffusion, so that the corrected values (denoted with an asterisk) satisfy the local equilibrium condition

$$
K_{\mathrm{A}}^{\mathrm{B}}=\frac{C_{\mathrm{A}}^{*} \cdot \overline{C_{\mathrm{B}}^{*}}}{\overline{C_{\mathrm{A}}^{*}} \cdot C_{\mathrm{B}}^{*}}
$$

Using the conditions of electroneutrality and material balance, this equation is converted to the form

$$
K_{\mathrm{A}}^{\mathrm{B}}=\frac{\left(C_{\mathrm{A}}+\Delta C_{\mathrm{A}}\right) \cdot\left(\overline{C_{\mathrm{B}}}+\Delta C_{\mathrm{A}} \cdot \frac{\delta}{\bar{\delta}}\right)}{\left(\overline{C_{\mathrm{A}}}-\Delta C_{\mathrm{A}} \cdot \frac{\delta}{\bar{\delta}}\right) \cdot\left(C_{\mathrm{B}}-\Delta C_{\mathrm{A}}\right)}
$$

where $C_{\mathrm{A}}$, and $C_{\mathrm{B}}$ are the concentrations of components $\mathrm{A}$ and $\mathrm{B}$ at the previous time point, $\Delta C_{\mathrm{A}}$ is the difference between the corrected and the initial value of the concentration of ion $\mathrm{A}, \delta$ is the thickness of the surface layer, and the horizontal upper bar denotes the membrane phase.

Equation 14 can be solved explicitly with respect to $\Delta C_{\mathrm{A}}$. Next, the corrected values of the concentrations of components $\mathrm{A}$ and $\mathrm{B}$ in the surface layers of both phases are calculated, and these values are further used to describe the diffusion processes in each phase in accordance with the difference form of the Fick equations (see the Supporting Information II for more details). Thus, while in the model considered in this work, the concentration of potentialdetermining ions in the surface layers of the solution and the membrane is calculated with the joint solution of three eqs 3 , 4 , and 7 , in the IET model, a phased account of ion exchange and diffusion processes is carried out. However, both models lead to identical results, which are in good agreement with the experimental data.

Figure 3 also shows that for sufficiently long measurement times, the curve calculated under the assumption of a constant concentration of the interfering ion in the bulk solution significantly deviates from the experimental data, so it is necessary to take into account not only the changes in the concentration of the interfering ion in the near-electrode layer, but also the changes in the bulk solution because of the interfering ion uptake by the membrane.

Figure 4 shows the dependencies of the potential on time for the nitrate-SE in mixed picrate and nitrate solutions for higher

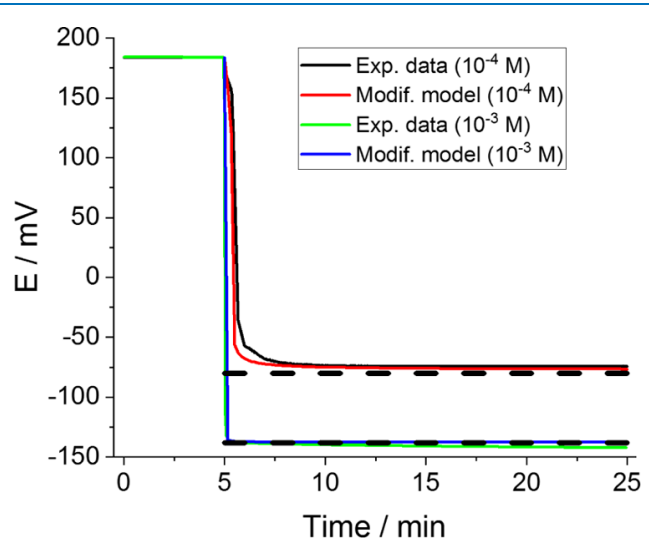

Figure 4. Dependencies of the potential vs time for nitrate-SE at high concentrations of picrate in the sample solution (indicated on the legend). The concentration of nitrate in both cases was $10^{-3} \mathrm{M}$. Simulation parameters are indicated in the caption to Figure 1. Dashed lines represent the potential calculated using the Nikolskii equation. 
concentrations of picrate, which confirms the high prognostic ability of the modified model in all real-life scenarios of ISE operation. The model predicts a very strong dependence of the potential on time and its sharp drop to the value predicted with the Nikolskii equation. The simulation results are in quite good agreement with the experimental data.

\section{CONCLUSIONS}

It is shown that the dynamic diffusion potential model proposed by Morf and improved by Bakker is not applicable in its current form to predict the functioning of ISEs in the presence of small concentrations of strongly interfering ions. The modification of this model, which consists in taking into account the concentration change of the interfering ion in the near-electrode layer of the solution, as well as the concentration change in the bulk solution, allows one to obtain an adequate description of the experimental results. This case, considering measuring in the presence of small concentrations of strongly interfering ions, seems to be quite realistic in some practical applications of ISEs. Therefore, the obtained results may be of practical interest for assessing the possibilities of performing measurements under certain experimental conditions, as well as for a priori search for ways to reduce the influence of the interfering ion by a targeted change in conditions affecting the intensity of diffusion that are amenable to regulation.

\section{EXPERIMENTAL DETAILS}

The object of the study was an ion exchanger-based nitrateselective electrode; the picrate ion was used as the interfering one. The choice was made for the reason of high lipophilicity of picrate ions and their guaranteed absence in nitrate salts, thus ensuring the purity of the experiment. ISE membranes were prepared using polyvinyl chloride as a binding polymer (20 wt \%), o-nitrophenyl octyl ether as a plasticizer (79.4 wt $\%)$, and tridodecylmethylammonium chloride as an ion exchanger $(0.6$ wt $\%$ or approximately $0.01 \mathrm{M})$. The unbiased selectivity coefficient $K_{\mathrm{NO}_{3} \text {, Pic }}^{\text {Pot }}=(3.8 \pm 0.2) \times 10^{5}$ was determined by the modified separate solution method. ${ }^{24}$ The study of the influence of the interfering ion on the ISE response was carried out in the following way. First, the electrodes were immersed into the initial solution of the primary ion $(20 \mathrm{~mL})$ for 15-20 min to achieve stable value of the potential (the potential drift did not exceed $0.2 \mathrm{mV} / \mathrm{min}$ ). After that, a certain volume $(2.2 \mathrm{~mL})$ of the previously prepared mixed solution containing an appropriate concentration of the interfering ion and the background of the primary ion corresponding to its concentration in the initial solution was added, preventing the interruption of the contact of the membrane with the solution, and the dynamics of the potential change was recorded. All the measurements were carried out at room temperature $19 \pm 1{ }^{\circ} \mathrm{C}$.

\section{SIMULATION DETAILS}

The following parameters were used in the simulation: the membrane thickness was $400 \mu \mathrm{m}$; the thicknesses of elementary layers in the both phases were $5 \mu \mathrm{m}$; and the time increment was $5 \mathrm{~ms}$. The area of the membrane was 1 $\mathrm{cm}^{2}$. Because in all experiments the main electrolyte in the sample solution was potassium nitrate, the diffusion coefficient of the ions in the solution was assumed to be equal to the arithmetic mean of the diffusion coefficients of potassium and nitrate ions $\left(1.6 \times 10^{-9} \mathrm{~m}^{2} / \mathrm{s}\right){ }^{25}$ The diffusion coefficient of ions in the membrane phase for a membrane containing $20 \mathrm{wt}$ $\%$ of PVC was chosen equal to $9 \times 10^{-12} \mathrm{~m}^{2} / \mathrm{s}$. The thickness of the water diffusion layers $(25 \mu \mathrm{m})$ was selected on the basis of experimental value of this parameter for a stirring rate of 500 rpm. ${ }^{26}$ The values of the potential were calculated according to the equation

$$
E=\text { const }-\frac{2.303 R T}{F} \log \frac{C_{\mathrm{A}, 0}(t)}{\overline{C_{\mathrm{A}, 0}(t)}}
$$

where const was chosen equal to the value at which the calculated ISE potential in the initial solution of the primary ion coincided with the experimental value (see the Supporting Information III,IV for the program code).

\section{ASSOCIATED CONTENT}

\section{S Supporting Information}

The Supporting Information is available free of charge on the ACS Publications website at DOI: 10.1021/acsomega.8b02926.

Comparison of diffusion-layer model proposed by Lewenstam and the Morf s-Bakker's model modified by us; description of the IET model; the code of the Morf s-Bakker's model modified by us (PDF)

\section{AUTHOR INFORMATION}

\section{Corresponding Author}

*E-mail: egorvv@bsu.by.

ORCID $\odot$

Vladimir V. Egorov: 0000-0001-9414-0423

Notes

The authors declare no competing financial interest.

\section{ACKNOWLEDGMENTS}

We acknowledge the CHEMREAGENTS program for financial support.

\section{REFERENCES}

(1) Mikhelson, K. N. Ion-Selective Electrodes; Springer: Berlin, Heidelberg, 2013; Vol. 81.

(2) Janata, J. Principles of Chemical Sensors; Springer: Heidelberg, 2009; pp 1-395.

(3) Sokalski, T.; Ceresa, A.; Zwickl, T.; Pretsch, E. Large improvement of the lower detection limit of ion-selective polymer membrane electrodes. J. Am. Chem. Soc. 1997, 119, 11347-11348.

(4) Mathison, S.; Bakker, E. Effect of transmembrane electrolyte diffusion on the detection limit of carrier-based potentiometric ion sensors. Anal. Chem. 1998, 70, 303-309.

(5) Ceresa, A.; Sokalski, T.; Pretsch, E. Influence of key parameters on the lower detection limit and response function of solvent polymeric membrane ion-selective electrodes. J. Electroanal. Chem. 2001, 501, 70-76.

(6) Szigeti, Z.; Vigassy, T.; Bakker, E.; Pretsch, E. Approaches to improving the lower detection limit of polymeric membrane ionselective electrodes. Electroanalysis 2006, 18, 1254-1265.

(7) Radu, A.; Peper, S.; Bakker, E.; Diamond, D. Guidelines for improving the lower detection limit of ion-selective electrodes: a systematic approach. Electroanalysis 2007, 19, 144-154.

(8) Hulanicki, A.; Lewenstam, A. Model for treatment of selectivity coefficients for solid-state ion-selective electrodes. Anal. Chem. 1981, 53, 1401-1405.

(9) Hulanicki, A.; Lewenstam, A. Variability of selectivity coefficients of solid-state ion-selective electrodes. Talanta 1982, 29, 671-674. 
(10) Maj-Zurawska, M.; Sokalski, T.; Hulanicki, A. Interpretation of the selectivity and detection limit of liquid ion-exchanger electrodes. Talanta 1988, 35, 281-286.

(11) Ilcheva, L.; Cammann, K. Selektivitätssteigerung ionenselektiver Elektroden durch kinetische Diskriminierung von Stôrionen. Fresenius' J. Anal. Chem. 1985, 320, 664.

(12) Paczosa-Bator, B.; Blaz, T.; Migdalski, J.; Lewenstam, A. Conducting polymers in modelling transient potential of biological membranes. Bioelectrochemistry 2007, 71, 66-74.

(13) Morf, W. E.; Pretsch, E.; De Rooij, N. F. Computer simulation of ion-selective membrane electrodes and related systems by finitedifference procedures. J. Electroanal. Chem. 2007, 602, 43-54.

(14) Bakker, E. Membrane response model for ion-selective electrodes operated by controlled-potential thin-layer coulometry. Anal. Chem. 2011, 83, 486-493.

(15) Bakker, E. Evaluation of Egorov's Improved Separate Solution Method for Determination of Low Selectivity Coefficients by Numerical Simulation. Anal. Chem. 2014, 86, 8021-8024.

(16) Kisiel, A.; Michalska, A.; Maksymiuk, K. Bilayer membranes for ion-selective electrodes. J. Electroanal. Chem. 2016, 766, 128-134.

(17) Egorov, V. V.; Novakovskii, A. D.; Zdrachek, E. A. Modeling of the effect of diffusion processes on the response of ion-selective electrodes by the finite difference technique: comparison of theory with experiment and critical evaluation. J. Anal. Chem. 2017, 72, 793802.

(18) Morf, W. E.; Pretsch, E.; de Rooij, N. F. Theory and computer simulation of the time-dependent selectivity behavior of polymeric membrane ion-selective electrodes. J. Electroanal. Chem. 2008, 614, $15-23$.

(19) Egorov, V. V.; Novakovskii, A. D.; Zdrachek, E. A. A simple dynamic diffusion model of the response of highly selective electrodes: the effect of simulation parameters and boundary conditions on the results of calculations. Russ. J. Electrochem. 2018, 54, 381-390.

(20) Egorov, V. V.; Novakovskii, A. D.; Zdrachek, E. A. An interface equilibria-triggered time-dependent diffusion model of the boundary potential and its application for the numerical simulation of the ionselective electrode response in real systems. Anal. Chem. 2018, 90, 1309-1316.

(21) Yuan, D.; Bakker, E. Overcoming pitfalls in boundary elements calculations with computer simulations of ion selective membrane electrodes. Anal. Chem. 2017, 89, 7828-7831.

(22) Jasielec, J. J. Modelling of Potentiometric Ion Sensors. Ph.D. Thesis, Åbo Akademi University, Finland, 2013.

(23) Bobacka, J.; Ivaska, A.; Lewenstam, A. Potentiometric ion sensors. Chem. Rev. 2008, 108, 329-351.

(24) Bakker, E. Determination of unbiased selectivity coefficients of neutral carrier-based cation-selective electrodes. Anal. Chem. 1997, 69, 1061-1069.

(25) Lange's Handbook of Chemistry; Dean, J. A., Ed.; McGraw-Hill: New York, 1998.

(26) Zdrachek, E. A.; Nazarov, V. A.; Egorov, V. V. The method of estimation of diffusion coefficients of ions in membranes of ionselective electrodes based on potentiometric data. Vestn. BGU, Ser. 2 2014, 1, 10-15. 